\title{
COMPARAÇÃO DE MÉTODOS DE AMOSTRAGEM EM FRAGMENTOS DE FLORESTA OMBRÓFILA MISTA, EM LEBON RÉGIS, SC
}

\author{
Saulo Jorge Téo ${ }^{1}$, Chaiane Rodrigues Schneider ${ }^{2}$, Luan Demarco Fiorentin ${ }^{3}$, Reinaldo Hoinacki da Costa ${ }^{4}$ \\ ${ }^{1}$ Eng. Florestal, M.Sc., UNOESC, Xanxerê, SC, Brasil - sauloteo@yahoo.com.br \\ ${ }^{2}$ Acadêmica de Eng. Florestal, UNOESC, Xanxerê, SC, Brasil - chai.rodriguesschneider@ gmail.com \\ ${ }^{3}$ Acadêmico de Eng. Florestal, UNOESC, Xanxerê, SC, Brasil - luanfiorentin@ hotmail.com \\ ${ }^{4}$ Eng. Florestal, Juliana Florestal Ltda., Caçador, SC, Brasil - reinaldo@ frameport.com.br \\ Recebido para publicação: 10/07/2013 - Aceito para publicação: 12/02/2014
}

\begin{abstract}
Resumo
O objetivo desta pesquisa foi avaliar e comparar métodos de amostragem de Área Fixa, Bitterlich, Strand e Prodan em fragmentos de Floresta Ombrófila Mista em Lebon Régis, SC. O processo de amostragem utilizada foi aleatória simples. Em cada unidade amostral, foram avaliadas todas as árvores de diâmetro à altura do peito maior ou igual a $5 \mathrm{~cm}$. Para o método de Área Fixa, utilizou-se dimensões 10 x $50 \mathrm{~m}\left(500 \mathrm{~m}^{2}\right)$; para Bitterlich e Strand, adotou-se Fator de Área Basal igual a 2; e para Prodan, foram avaliadas as 6 árvores mais próximas. Apenas o método de amostragem da Área Fixa apresentou suficiência amostral, porém também apresentou o maior tempo de execução no campo, com resultados significativamente diferentes dos demais. Já a eficiência relativa apresentou-se maior para o método de Strand, porém sem diferença estatística significativa com relação aos demais. Não houve diferenças significativas entre o número de árvores e área basal por hectare, estimados pelos diferentes métodos de amostragem.

Palavras-chave: Inventário Florestal; eficiência relativa; floresta com araucária.
\end{abstract}

\begin{abstract}
Comparison of sampling methods in Mixed Ombrophyllous Forest fragments, in Lebon Régis - SC. The objective of this research was to evaluate and compare sampling methods Fixed Area, Bitterlich, Strand and Prodan, in fragments of Mixed Ombrophyllous Forest, in Lebon Régis, SC state, Brazil. The forest inventory applies a simple random sampling. At each sampling unit, we evaluated every tree with diameter at breast height greater than or equal to $5 \mathrm{~cm}$. For the method of Fixed Area we used dimensions of $10 \times 50 \mathrm{~m}\left(500 \mathrm{~m}^{2}\right)$, for Bitterlich and Strand we adopted Basal Area Factor equal to 2, and for Prodan we evaluated the 6 nearest trees. Only the Fixed Area method presented sampling sufficiency, however, it presented highest execution time in the field as well, with results significantly different from the other sampling methods. The relative efficiency was higher for Strand, but without statistical significance compare to the other methods. There were no significant differences between the number of trees and basal area per hectare, estimated by the different sampling methods.
\end{abstract}

Keywords: Forest inventory; relative efficiency; araucaria forest.

\section{INTRODUÇÃO}

A denominação Floresta Ombrófila Mista é utilizada pelo Instituto Brasileiro de Geografia e Estatística (IBGE) na classificação fisionômico-ecológica da vegetação brasileira. Pertencente ao Bioma da Mata Atlântica, que abriga grande parte da diversidade biológica do Brasil, essa tipologia florestal ocorre nos planaltos da região Sul, situados a oeste da Serra do Mar, havendo elevado número de espécies ameaçadas de extinção (SERVIÇO FLORESTAL BRASILEIRO, 2010). Sua estrutura estratificada possui formações com dosséis uniformes e emergentes, caracterizados unicamente pelas copas da Araucaria angustifolia (Bert.) O. Ktze. (MARTINS, 2009).

Contudo, diversas atividades antrópicas iniciadas no século XX provocaram a dramática redução das áreas de florestas originais na região, agravando-se pela carência de espaços legalmente protegidos 
(MEDEIROS et al., 2005). Por isso, defender esses remanescentes é o desafio atual, necessitando medidas de caráter científico, para facilitar a efetivação de práticas conservacionistas e de manejo (SANQUETTA, 2001).

Nesse sentido, os inventários florestais são de grande contribuição na caracterização de florestas, em especial as nativas, valorizando seu conhecimento e uso sustentável, isso porque são definidos como o estudo, desenvolvimento e aplicação de técnicas para quantificar e qualificar a vegetação (PÉLLICO NETTO; BRENA, 1997). O início de atividades de manejo, qualquer que seja a finalidade, deve ser precedido de um inventário, o que reforça o desenvolvimento da ciência, dispondo de técnicas a serem aplicadas, compostas por diferentes sistemas, processos e métodos de amostragem.

O método de amostragem de Área Fixa destaca-se por ser o mais antigo e conhecido, sendo a seleção das árvores feita de acordo com a frequência em que estão dispostas na floresta e com o tamanho da unidade amostral (SANQUETTA et al., 2006). Já o método de Bitterlich consiste em contar as árvores, em um giro de $360^{\circ}$, cujos diâmetros à altura do peito $(d)$ são iguais ou maiores a uma abertura angular, selecionando as árvores com probabilidade proporcional à área basal (PÉLLICO NETTO; BRENA, 1997).

$\mathrm{O}$ método de Strand fundamenta-se em um critério probabilístico de seleção das árvores proporcional ao diâmetro, para o cálculo da área basal e número de árvores por hectare; e proporcional à altura, para obter o volume e número de árvores por hectare (PÉLLICO NETTO; BRENA, 1997). Diferentemente do método de Bitterlich, a unidade amostral de Strand é composta por uma linha de amostragem.

O método de Prodan, também conhecido como método das seis árvores, envolve parcelas de área variável, selecionando-se as seis árvores mais próximas ao ponto central da unidade amostral, realizando-se a inclusão dos indivíduos proporcionalmente à sua distância a um ponto de origem (PRODAN et al., 1997).

Dentre os métodos mais utilizados, destaca-se o da área fixa, enquanto os demais raramente são empregados. Alguns trabalhos foram realizados, ao longo dos anos, sobre a comparação de diferentes métodos de amostragem para obtenção de estimativas dos parâmetros quantitativos e qualitativos de diferentes tipologias florestais (MOSCOVICH et al., 1999; VANINI, 1999; GORENSTEIN, 2002; AGUIAR, 2003; DIAS; COUTO, 2005), porém ainda são insuficientes para determinar a qualidade das estimativas obtidas desses parâmetros, bem como a eficiência e operacionalidade de campo em inventários, sobretudo para Floresta Ombrófila Mista. Por esse fato, decidir pelo método de amostragem mais adequado para cada tipologia florestal é tema de grande relevância para a ciência florestal.

Dias (2005), na avaliação da composição florística e fitossociológica, por meio de diferentes métodos de amostragem, em uma Floresta Ombrófila Densa do Parque Estadual Carlos Botelho, SP, encontrou que os métodos de Área Fixa e Bitterlich apresentaram maior heterogeneidade em nível de família e determinação da dominância $\left(\mathrm{m}^{2} / \mathrm{ha}\right)$. Porém a densidade (árv./ha) da floresta foi mais bem avaliada pelos métodos de Área Fixa e Quadrantes.

Druszcz et al. (2012) compararam o inventário florestal de um plantio de Pinus taeda L. realizado pelo método da Área Fixa com o método de Bitterlich, avaliando a precisão, eficiência relativa e custos da estimativa de diferentes variáveis. Ao final da pesquisa, recomendou-se o método da Área Fixa para estimativa do número de árvores por unidade de área e o método de Bitterlich para estimativa das variáveis diâmetro médio, área basal e volume por unidade de área.

Moscovich et al. (1999), em área de Floresta Ombrófila Mista, compararam os métodos de amostragem de Área Fixa, Bitterlich, Strand, Prodan e Quadrantes. Os autores concluíram, de acordo com a eficiência relativa, que Strand foi o método que se apresentou mais eficiente para estimativa dos parâmetros volume comercial com casca, área basal, número de árvores por hectare e abrangência de espécies.

Este trabalho teve como objetivos a análise e comparação da suficiência amostral, composição florística, estimadores de número de árvores e área basal por hectare, tempo de execução e eficiência relativa para os métodos de amostragem de Área Fixa, Bitterlich, Strand e Prodan, em fragmentos de Floresta Ombrófila Mista localizados no município de Lebon Régis, Santa Catarina.

\section{MATERIAL E MÉTODOS}

A área de estudo está localizada na cidade de Lebon Régis, Santa Catarina, especificamente na Fazenda São Sebastião, a qual, com a inclusão de áreas de plantio de Pinus taeda L. e fragmentos de 
Floresta Ombrófila Mista, possui 392,95 hectares de extensão, situada na região centro-oeste do estado, pertencente à empresa Juliana Florestal Ltda., de Caçador, SC (Figura 1).

A vegetação original da área é a Floresta Ombrófila Mista, em sua formação Floresta Ombrófila Mista Montana, a qual ocupava quase inteiramente o planalto acima de $500 \mathrm{~m}$ de altitude, nos estados do Paraná, Santa Catarina e Rio Grande do Sul (IBGE, 2012).

O clima é $\mathrm{Cfb}$, ou seja, mesotérmico úmido, sem estação seca, com verões frescos e apresentando invernos rigorosos com geadas severas, conforme a classificação climática de Köppen. Conforme informações meteorológicas da Estação Experimental de Caçador, a temperatura média anual é de $16,5^{\circ} \mathrm{C}$, a precipitação média anual é de $1.608 \mathrm{~mm}$, a umidade relativa do ar média anual é de $77 \%$ e há ocorrência de, em média, 26 geadas por ano (KURASZ, 2005).

Os principais solos da área de estudo são Cambissolos háplicos, com grande variação de profundidade e drenagem variando de acentuada à imperfeita, e Nitossolos brunos, de textura argilosa ou muito argilosa, em geral moderadamente ácidos a ácidos (IBGE, 2007).

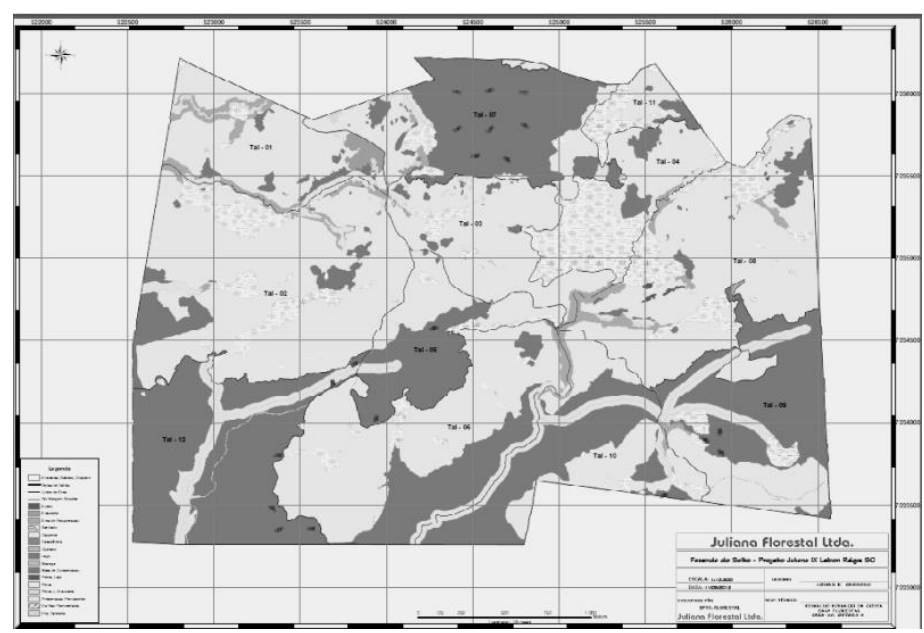

Figura 1. Área de estudo com os fragmentos de Floresta Ombrófila Mista representados em cinza claro, em Lebon Régis, SC.

Figure 1. Focused area with the Mixed Ombrophyllous Forest fragments represented in light gray, in Lebon Régis, SC.

As 20 unidades amostrais foram distribuídas aleatoriamente na área de estudo (Figura 1), sendo que a instalação de cada amostra, para todos os métodos, procedeu de um mesmo ponto de origem, deixando-as igualmente localizadas. Na floresta, foram identificadas e medidas as árvores com diâmetro à altura do peito $(d)$ igual ou maior a $5 \mathrm{~cm}$.

A identificação das espécies encontradas procedeu-se com ajuda de um mateiro e coleta do material botânico, o qual foi analisado com auxílio da literatura e do herbário do Departamento de Ciências Florestais da Universidade Federal do Paraná, localizado em Curitiba, Paraná.

No levantamento dos dados de campo, para o método de amostragem de Área Fixa, foram medidas unidades amostrais de forma retangular, com dimensões de 10 x $50 \mathrm{~m}\left(500 \mathrm{~m}^{2}\right)$ (SANQUETTA et al., 2006). Para Bitterlich e Strand, com uso do relascópio de espelho de Bitterlich, adotou-se Fator de Área Basal igual a $2(\mathrm{FAB}=2)$. Adicionalmente, para o método de Strand, o comprimento da unidade amostral foi de $15,71 \mathrm{~m}$, o equivalente a $5 \pi$. Para o método de Prodan, o raio da sexta árvore foi medido e tomado como referência para os cálculos dos estimadores das unidades amostrais.

Os procedimentos foram realizados com uso dos seguintes equipamentos: trena de agrimensor $(50 \mathrm{~m})$, trena de $30 \mathrm{~m}$, fitas métricas, balizas e relascópio de espelho de Bitterlich. Para adequação do material botânico coletado no campo, o uso de uma prensa e etiquetas de identificação foi indispensável.

A suficiência amostral pode ser realizada de acordo com a área amostrada, porém neste estudo, como se trata não somente do método de amostragem de Área Fixa, mas também de métodos de área variável (Bitterlich, Strand e Prodan), analisou-se a suficiência amostral de acordo com a representação 
gráfica do número de espécies contra o número de unidades amostrais, para cada método de amostragem (MUELLER-DOMBOIS; ELLEMBERG, 1974).

Para a obtenção da eficiência relativa, adotou-se o cálculo proposto por Freese (1967). Antes do cálculo da eficiência relativa, foi necessário tomar o tempo médio de instalação e medição das unidades amostrais, medido com uso de um cronômetro digital, e calcular o coeficiente de variação para o número de árvores e área basal por hectare. O cálculo do coeficiente de variação e eficiência relativa foram feitos como segue:

$$
\mathrm{CV}=\frac{\mathrm{s}_{\mathrm{x}}}{\overline{\mathrm{x}}} * 100 \quad \mathrm{ER}=\frac{1}{\mathrm{t} * \mathrm{CV}^{2}}
$$

em que: $\mathrm{CV}=$ coeficiente de variação;

$\mathrm{s}_{\mathrm{x}}=$ desvio padrão número de árvores e área basal por hectare;

$\overline{\mathrm{x}}=$ média do número de árvores e área basal por hectare;

$\mathrm{ER}=$ eficiência relativa;

$\mathrm{t}=$ tempo médio em minutos para cada método de amostragem.

Posteriormente, efetuou-se o teste de homogeneidade das variâncias e a análise de variância, com uso do programa computacional MS Excel ${ }^{\circledR}$. Considerou-se o delineamento inteiramente casualizado no processamento dos dados, em que os métodos de amostragem constituíram os tratamentos, e os valores obtidos para cada unidade amostral, as repetições. Essa análise estatística foi feita para o número de árvores por hectare, área basal por hectare, tempo de instalação e medição das unidades amostrais e eficiência relativa. Na presença de diferenças significativas pelo teste de $F(\alpha=5 \%)$ entre esses valores estimados, efetuou-se o teste de comparação de médias de Tukey $(\alpha=5 \%)$, para análise dessas diferenças.

\section{RESULTADOS E DISCUSSÃO}

Na figura 2 é apresentada a suficiência amostral obtida para os diferentes métodos de amostragem avaliados.
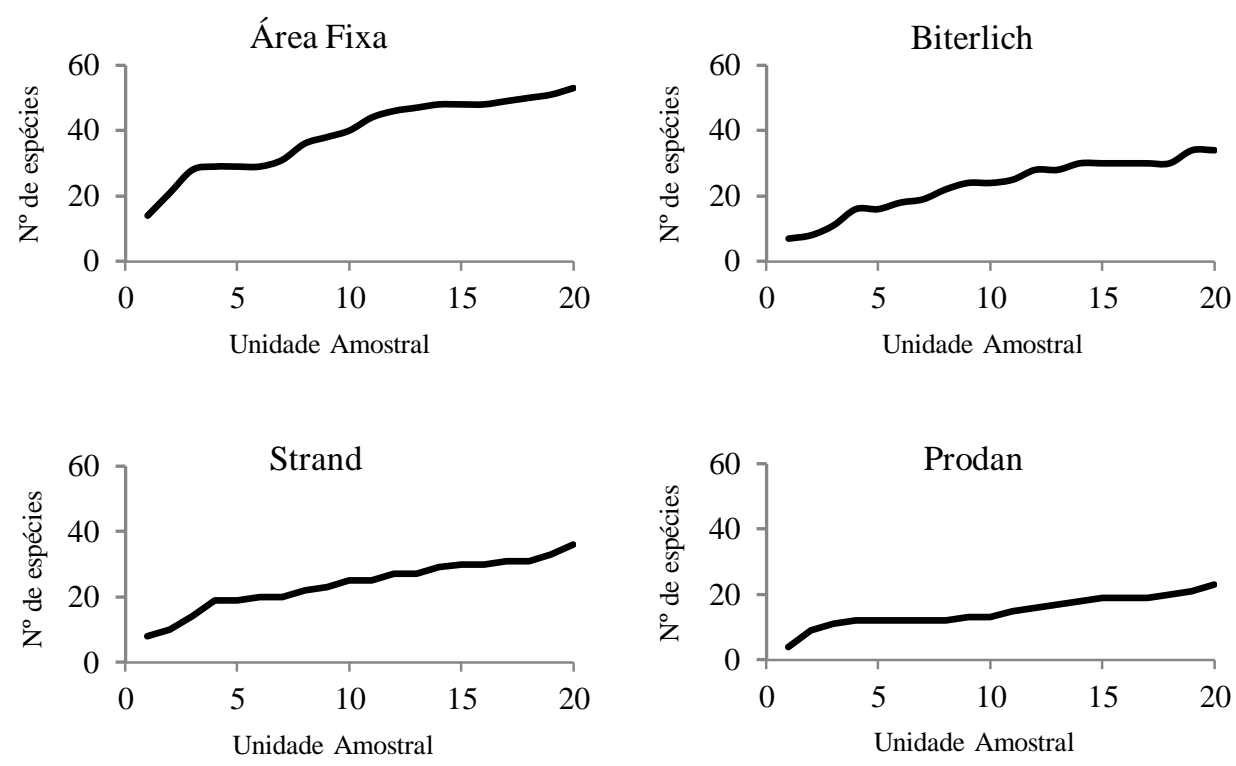

Figura 2. Relação entre o número de espécies e unidades amostrais para os métodos de amostragem de Área Fixa, Bitterlich, Strand e Prodan, em fragmentos de Floresta Ombrófila Mista, Lebon Régis, SC.

Figure 2. Relation between the number of species and sampling units for the sampling of Fixed Area, Bitterlich, Strand and Prodan in fragments of Ombrophyllous Forest, Lebon Régis, SC. 
Diante da figura 2, é possível perceber que, para todos os métodos de amostragem avaliados, os gráficos apresentaram oscilações, sem tendência clara à estabilização do número de espécies com relação ao número de unidades amostrais. A medição de novas unidades amostrais poderia proporcionar uma relação espécie/unidade amostral com estabilização mais nítida.

Nos métodos de amostragem de Área Fixa e Bitterlich houve tendência à estabilização do número de espécies amostradas, porém ele torna a aumentar a partir da $16^{\circ}$ e $18^{\circ}$ unidade amostral, respectivamente. Para o método da Área Fixa, embora também tenha ocorrido um aumento do número de espécies amostradas nas últimas unidades amostrais, esse aumento não foi acentuado, e o número de espécies permanece em torno de 50 desde a parcela número 12. Por outro lado, quando são analisados os gráficos para os métodos de amostragem de Strand e Prodan, vê-se uma tendência crescente do número de espécies com o aumento do número de unidades amostrais.

Além disso, o número de espécies amostradas nesse estudo é superior ao encontrado por Negrelle e Silva (1992), para a mesma tipologia florestal. No entanto, Herrera et al. (2009) reportaram que a suficiência amostral para um levantamento florístico e análise fitossociológica da reserva florestal da Empresa Brasileira de Pesquisa Agropecuária e Empresa de Pesquisa Agropecuária e Extensão Rural de Santa Catarina(EMBRAPA/EPAGRI) (Floresta Ombrófila Mista), em Caçador, SC, foi de 42 parcelas, totalizando $20.000 \mathrm{~m}^{2}$ de área medida, com a identificação de cerca de 70 espécies botânicas.

Adicionalmente, além de analisar a figura 2 e considerar que as 20 unidades amostrais equivalem, para o método de amostragem de Área Fixa, a 1 hectare de área amostrada, a comparação com os trabalhos para suficiência amostral apresentou similaridades. Na pesquisa de Cordeiro e Rodrigues (2007), a suficiência resultou em 45 espécies amostradas em 0,3 ha; em Lingner et al. (2007), 41 identificações em 2,5 ha; em Martins et al. (2012), cerca de 50 espécies em 0,6 ha; em Nascimento et al. (2001), 54 espécies em 20 unidades amostrais; em Negrelle e Silva (1992), 43 espécies em 70 pontos de amostragem; e em Seger et al. (2005), 44 espécies em 15 unidades amostrais. Dessa forma, é possível afirmar semelhanças nos resultados de suficiência amostral em Floresta Ombrófila Mista, com relação ao número de espécies amostradas para o método da Área Fixa. Entretanto, o mesmo não ocorreu para os métodos de amostragem de Bitterlich, Strand e Prodan.

A tabela 1 apresenta, para cada método de amostragem avaliado, as espécies e as respectivas famílias a que pertencem os fragmentos estudados. O método de Área Fixa amostrou o maior número de espécies (53), seguido pelo método de amostragem de Strand (36), Bitterlich (34) e Prodan (23).

Ao todo, foram 15 as espécies florestais amostradas somente pelo método da Área Fixa, ou seja, pelos demais métodos não foram amostradas: Allphylus edulis, Annona crassiflera, Brunfelsia pilosa, Calyptranthes concinna, Campomanesia xanthocarpa, Cedrella fissilis, Celtis iguanaea, Conyza canadenses, Maytenus iliciolia, Myrcia obtecta, Myrsine coriacea, Nectandra megapotamica, Rhamnus sphaerosperma, Swartzia langsdorffii e Ziziphus joazeiro. Por outro lado, 20 espécies foram amostradas por todos os métodos de amostragem avaliados.

Da mesma forma ocorre com as famílias botânicas correspondentes a essas espécies. As famílias Aquifoliaceae, Araucariaceae, Asteraceae, Bignoniaceae, Canellaceae, Euphorbiaceae, Fabaceae, Lauraceae, Myrsinaceae, Myrtaceae, Proteaceae, Rosaceae, Rutaceae, Salicaceae, Stiracaceae e Winteraceae foram amostradas em todos os métodos de amostragem, porém famílias como Annonaceae, Meliaceae, Rhamnaceae e Ulmaceae só foram amostradas no método de amostragem de Área Fixa.

Moscovich et al. (1999) compararam os métodos de amostragem de Área Fixa, Bitterlich, Strand, Prodan e Quadrantes e obtiveram uma abrangência de espécies semelhante, porém maior para o método de Área Fixa. Para os demais métodos, houve grande percentagem da espécie Araucaria angustifolia, com pouca representação das demais espécies. Os autores justificaram que os métodos de amostragem de Bitterlich, Strand, Prodan e Quadrantes amostraram as espécies mais representativas da fitossociologia da floresta.

Para Dias e Couto (2005), a diferença entre o número de espécies amostradas pelos métodos deve-se principalmente ao número de indivíduos amostrados em cada método. Os autores obtiveram maior número de indivíduos amostrados pelo método de Área Fixa e o menor para o método de Bitterlich e dos Quadrantes, utilizando 64 unidades amostrais. O mesmo acontece para o número de espécies amostradas, o que sugere a realização de um número maior de unidades amostrais para os métodos de amostragem de Bitterlich e dos Quadrantes. 
Aguiar (2003), na comparação entre os métodos da Área Fixa e dos quadrantes para caracterização de composição florística e fitossociológica de um trecho de Floresta Ombrófila Densa, ressaltou que os métodos amostraram a composição florística da vegetação estudada de maneira semelhante, principalmente quando se consideraram as famílias, gêneros e espécies. Porém o método de Quadrantes amostrou 70,24\% do total de espécies levantadas pelo método da Área Fixa. O mesmo ocorre quando analisada a intensidade amostral: para Área Fixa, o número de espécies estabilizou a partir da unidade 35, enquanto que para Quadrantes, na unidade 26.

Vanini (1999) comparou os métodos de Área Fixa e de Bitterlich para realização de levantamento fitossociológico em três locais de Floresta Ombrófila Densa permanentemente alagada (caixetais) no município de Iguape, SP. Foram feitas 42 e 46 unidades amostrais, para o método da Área Fixa e de Bitterlich, respectivamente. De acordo com a autora, o método de Bitterlich, com fator de área basal igual a $1(\mathrm{FAB}=1)$, foi capaz de amostrar o mesmo número de espécies que o método da área fixa, porém utilizando apenas $1 / 3$ do tempo.

Tabela 1. Famílias e as respectivas espécies amostradas para cada método de amostragem avaliado, em fragmentos de Floresta Ombrófila Mista, em Lebon Régis, SC.

Table 1. Families and their species sampled for each sampling method evaluated, for the fragments of Ombrophyllous Mixed Forest, in Lebon Régis, SC.

\begin{tabular}{|c|c|c|c|c|c|}
\hline FAMÍLIA & Espécie & $\mathbf{A F}$ & B & $\mathbf{S}$ & $\mathbf{P}$ \\
\hline ANACARDIACEAE & Lithraea brasiliensis Marchand & $\mathrm{X}$ & $\mathrm{X}$ & $\mathrm{X}$ & $\mathrm{X}$ \\
\hline ANNONACEAE & Annona crassiflora Mart. & $\mathrm{X}$ & & & \\
\hline \multirow{3}{*}{ AQUIFOLIACEAE } & Ilex brevicuspis Reissek & $\mathrm{X}$ & & $\mathrm{X}$ & \\
\hline & Ilex paraguariensis A. St.-Hil. & $\mathrm{X}$ & $\mathrm{X}$ & $\mathrm{X}$ & $\mathrm{X}$ \\
\hline & Ilex theazans Mart. & $\mathrm{X}$ & $\mathrm{X}$ & $\mathrm{X}$ & $\mathrm{X}$ \\
\hline ARAUCARIACEAE & Araucaria angustifolia (Bertol.) Kuntze & $\mathrm{X}$ & $\mathrm{X}$ & $\mathrm{X}$ & $\mathrm{X}$ \\
\hline \multirow[t]{3}{*}{ ASTERACEAE } & Conyza canadensis (L.) Cronquist & $\mathrm{X}$ & & & \\
\hline & Gochnatia polymorpha (Lessing) Cabrera & $\mathrm{X}$ & $\mathrm{X}$ & $\mathrm{X}$ & $\mathrm{X}$ \\
\hline & Piptocarpha angustifolia Dusén ex Malme & $\mathrm{X}$ & $\mathrm{X}$ & $\mathrm{X}$ & \\
\hline BIGNONIACEAE & Jacaranda puberulla Chamisso & $\mathrm{X}$ & $\mathrm{X}$ & $\mathrm{X}$ & $\mathrm{X}$ \\
\hline CANELLACEAE & Cinnamodendron dinisii (Schwacke) Occhioni & $\mathrm{X}$ & $\mathrm{X}$ & $\mathrm{X}$ & $\mathrm{X}$ \\
\hline \multirow[t]{2}{*}{ CELASTRACEAE } & Citronella gongonha (Mart.) R. A. Howard & $\mathrm{X}$ & $\mathrm{X}$ & $\mathrm{X}$ & \\
\hline & Maytenus iliciolia (Schrad.) Planch. & $\mathrm{X}$ & & & \\
\hline \multirow[t]{2}{*}{ CUNONIACEAE } & Lamanonia ternata Vell. & $\mathrm{X}$ & $\mathrm{X}$ & $\mathrm{X}$ & \\
\hline & Weinmannia humilis Engl. & $\mathrm{X}$ & $\mathrm{X}$ & & $\mathrm{X}$ \\
\hline \multirow[t]{2}{*}{ EUPHORBIACEAE } & Sapium glandulatum (Vell.) Pax & $\mathrm{X}$ & $\mathrm{X}$ & $\mathrm{X}$ & \\
\hline & Sebastiania commersoniana L.B. Sm. \& Downs & $\mathrm{X}$ & $\mathrm{X}$ & $\mathrm{X}$ & $\mathrm{X}$ \\
\hline \multirow[t]{3}{*}{ FABACEAE } & Inga lentiscifolia Benth. & $\mathrm{X}$ & $\mathrm{X}$ & $\mathrm{X}$ & $\mathrm{X}$ \\
\hline & Mimosa scabrella Benth. & $\mathrm{X}$ & $\mathrm{X}$ & $\mathrm{X}$ & $\mathrm{X}$ \\
\hline & Swartzia langsdorffii Raddi & $\mathrm{X}$ & & & \\
\hline \multirow[t]{4}{*}{ LAURACEAE } & Nectandra megapotamica (Spreng.) Mez & $\mathrm{X}$ & & & \\
\hline & Ocotea elegans $\mathrm{Mez}$ & $\mathrm{X}$ & $\mathrm{X}$ & $\mathrm{X}$ & $\mathrm{X}$ \\
\hline & Ocotea porosa (Nees \& C. Mart.) Barroso & $\mathrm{X}$ & $\mathrm{X}$ & $\mathrm{X}$ & $\mathrm{X}$ \\
\hline & Persea pyrifolia Nees & $\mathrm{X}$ & $\mathrm{X}$ & $\mathrm{X}$ & \\
\hline MELIACEAE & Cedrella fissilis Vellozo & $\mathrm{X}$ & & & \\
\hline \multirow[t]{2}{*}{ MYRSINACEAE } & Myrsine coriacea (Sw.) Roem. \& Schult. & $\mathrm{X}$ & & & \\
\hline & Myrsine gardneriana DC. & $\mathrm{X}$ & $\mathrm{X}$ & $\mathrm{X}$ & $\mathrm{X}$ \\
\hline \multirow[t]{8}{*}{ MYRTACEAE } & Acca sellowiana (O. Berg) Burret & $\mathrm{X}$ & $\mathrm{X}$ & $\mathrm{X}$ & \\
\hline & Calyptranthes concinna DC. & $\mathrm{X}$ & & & \\
\hline & Campomanesia xanthocarpa O. Berg & $\mathrm{X}$ & & & \\
\hline & Eugenia handroana D. Legrand & $\mathrm{X}$ & $\mathrm{X}$ & & $\mathrm{X}$ \\
\hline & Myrcia guianensis (Aubl.) DC. & $\mathrm{X}$ & $\mathrm{X}$ & $\mathrm{X}$ & $\mathrm{X}$ \\
\hline & Myrcia obtecta (O. Berg) Kiaersk. & $\mathrm{X}$ & & & \\
\hline & Myrcia palustres DC. & $\mathrm{X}$ & & $\mathrm{X}$ & \\
\hline & Myrciaria tenella (DC.) O. Berg & $\mathrm{X}$ & $\mathrm{X}$ & $X$ & \\
\hline
\end{tabular}




\begin{tabular}{|c|c|c|c|c|c|}
\hline PHYTOLACCACEAE & Seguieria langsdorffii Moq. & $\mathrm{X}$ & $\mathrm{X}$ & $X$ & \\
\hline PROTEACEAE & Roupala brasiliensis Klotzsch & $\mathrm{X}$ & $\mathrm{X}$ & $\mathrm{X}$ & $\mathrm{X}$ \\
\hline \multirow[t]{2}{*}{ RHAMNACEAE } & Rhamnus sphaerosperma $\mathrm{Sw}$. & $\mathrm{X}$ & & & \\
\hline & Ziziphus joazeiro Mart. & $\mathrm{X}$ & & & \\
\hline ROSACEAE & Prunus myrtifolia (L.) Urb. & $\mathrm{X}$ & $\mathrm{X}$ & $\mathrm{X}$ & $\mathrm{X}$ \\
\hline RUBIACEAE & Coutarea hexandra (Jacq.) K. Schum & $\mathrm{X}$ & & $\mathrm{X}$ & \\
\hline \multirow[t]{2}{*}{ RUTACEAE } & Metrodorea nigra St.-Hil & $\mathrm{X}$ & $\mathrm{X}$ & $\mathrm{X}$ & $\mathrm{X}$ \\
\hline & Zanthoxylum rhoifolium Lam. & $\mathrm{X}$ & $\mathrm{X}$ & $\mathrm{X}$ & \\
\hline \multirow[t]{2}{*}{ SALICACEAE } & Casearia decandra Jacq. & $\mathrm{X}$ & $\mathrm{X}$ & $\mathrm{X}$ & $\mathrm{X}$ \\
\hline & Xylosma ciliatifolia (Clos) Eichler & $\mathrm{X}$ & $\mathrm{X}$ & $\mathrm{X}$ & \\
\hline \multirow[t]{2}{*}{ SAPINDACEAE } & Allophylus edulis (Cambess. \& A. Juss.) Radlk. & $\mathrm{X}$ & & & \\
\hline & Matayba elaeagnoides Radlk. & $\mathrm{X}$ & & $\mathrm{X}$ & $\mathrm{X}$ \\
\hline \multirow[t]{2}{*}{ SOLANACEAE } & Brunfelsia pilosa (Plowman) & $\mathrm{X}$ & & & \\
\hline & Solanum mauritianum Scop. & $\mathrm{X}$ & $\mathrm{X}$ & $\mathrm{X}$ & \\
\hline STIRACACEAE & Styrax leprosus Hook. \&Arn. & $\mathrm{X}$ & $\mathrm{X}$ & $\mathrm{X}$ & $\mathrm{X}$ \\
\hline SYMPLACACEAE & Symplocos uniflora (Pohl.) Benth. & $\mathrm{X}$ & $\mathrm{X}$ & $\mathrm{X}$ & \\
\hline ULMACEAE & Celtis iguanaea (Jacq.) Sargent & $\mathrm{X}$ & & & \\
\hline WINTERACEAE & Drimys brasiliensis Miers & $\mathrm{X}$ & $\mathrm{X}$ & $\mathrm{X}$ & $\mathrm{X}$ \\
\hline
\end{tabular}

AF: método de amostragem de Área Fixa; B: método de amostragem de Bitterlich; S: método de amostragem de Strand; P: método de amostragem de Prodan.

Na tabela 2, é possível analisar os resultados obtidos para o número de árvores por hectare, área basal por hectare e o tempo por parcela obtido para cada método a partir dos valores da média, das 20 unidades amostrais. Adicionalmente, estão os resultados dos coeficientes de variação obtidos para o número de árvores e área basal por hectare.

Tabela 2. Resultados estimados para cada método de amostragem para número de árvores por hectare, área basal, coeficiente de variação e tempo médio de instalação e medição das unidades amostrais, para fragmentos de Floresta Ombrófila Mista, em Lebon Régis, SC.

Table 2. Estimated results for each sampling method for the number of trees per hectare, basal area, coefficient of variation and installation and measurement time of sample units, for the fragments of Mixed Ombrophyllous Forest, in Lebon Régis, SC.

\begin{tabular}{lcccc}
\hline \multirow{2}{*}{ Variável } & \multicolumn{3}{c}{ Método de amostragem } \\
\cline { 2 - 5 } & Área Fixa & Bitterlich & Strand & Prodan \\
\hline $\mathrm{N}($ árv/ha) & $1703 \mathrm{~ns}$ & $2511 \mathrm{~ns}$ & $2495 \mathrm{~ns}$ & $2311 \mathrm{~ns}$ \\
$\mathrm{CV}(\%)$ & 24,29 & 42,99 & 46,28 & 60,23 \\
$\mathrm{G}\left(\mathrm{m}^{2} / \mathrm{ha}\right)$ & $35,00 \mathrm{~ns}$ & $45,60 \mathrm{~ns}$ & $40,00 \mathrm{~ns}$ & $33,09 \mathrm{~ns}$ \\
$\mathrm{CV}(\%)$ & 23,79 & 24,05 & 24,54 & 50,69 \\
Tempo médio (min/ua) & $49,55 \mathrm{a}$ & $17,88 \mathrm{~b}$ & $9,67 \mathrm{c}$ & $5,89 \mathrm{~d}$ \\
\hline
\end{tabular}

Nota: N: número de árvores por hectare; G: área basal por hectare; ua: unidade amostral; ns: não significativo para teste de $F$ ( $\alpha$ : $5 \%$ ), na análise de variância. Valores seguidos de letras diferentes apresentaram diferenças significativas segundo o teste de Tukey $(\alpha: 5 \%)$.

Conforme os resultados apresentados na tabela 2, o método de amostragem de Bitterlich apresentou o maior número de árvores e área basal por hectare, e os menores valores foram obtidos para os métodos de Área Fixa e Prodan, entretanto essas diferenças não foram estatisticamente significativas. Para Farias et al. (2002), na comparação de métodos de amostragem de Área Fixa e Bitterlich de Floresta Estacional Semidecidual, em Viçosa, MG, as variáveis área basal e número de árvores por hectare não apresentaram diferenças significativas entre os métodos de amostragem.

Portanto, quando avaliados os coeficientes de variação para número de árvores por hectare e área basal por hectare, obteve-se menor variabilidade para o método de amostragem de Área Fixa, e maior para o método de amostragem de Prodan. Para os métodos de amostragem de Bitterlich e Strand, observou-se variabilidade semelhante. Esse resultado pode ser atribuído à forma de inclusão das árvores e pelo tamanho da unidade amostral de cada método. 
Dias e Couto (2005) relataram que o número de árvores por hectare foi melhor avaliado pelos métodos de Área Fixa e Quadrantes. Já para área basal, os métodos da Área Fixa, Quadrantes e Bitterlich apresentaram melhores estimativas. No entanto, Druszcz et al. (2010), quando compararam os métodos de Área Fixa e Bitterlich em plantio de Pinus taeda, não encontraram diferenças significativas entre os métodos de amostragem quando avaliados o número de árvores por hectare e área basal por hectare.

O método de amostragem de Área Fixa apresentou o maior tempo de execução do inventário, seguido dos métodos de amostragem de Bitterlich, Strand e Prodan. Todos os métodos de amostragem, para o tempo de instalação e execução, apresentaram diferenças significativas em suas médias, quando analisadas pelo teste de Tukey, com nível de significância de 5\% $(\alpha=0,05)$ (Tabela 2).

Vanini (1999), na comparação dos métodos de Área Fixa e de Bitterlich em Floresta Ombrófila Densa permanentemente alagada (caixetais), concluiu que o método de amostragem de Bitterlich se mostrou mais eficiente que o método de Área Fixa, uma vez que não ocorreram problemas na visualização das árvores e o deslocamento facilitado permitiu maior coleta de informações sobre a floresta.

De acordo com a tabela 3, o método que apresentou maior eficiência relativa, tanto na determinação do número de árvores por hectare quanto na estimativa de área basal foi o método de amostragem de Strand. Essa informação coincide com os resultados obtidos por Moscovich et al. (1999), em que o método de Strand mostrou-se mais eficiente para estimar área basal e número de árvores por hectare. Entretanto, de acordo com a análise de variância, os métodos de amostragem não apresentaram diferenças significativas para a eficiência relativa calculada para as diferentes estimativas dos parâmetros da população estudada.

Tabela 3. Eficiência relativa para os métodos de amostragem de Área Fixa, Bitterlich, Strand e Prodan, para estimativa do número de árvores e área basal, em fragmentos de Floresta Ombrófila Mista em Lebon Régis, SC.

Table 3. Relative efficiency to the sampling methods of Fixed Area, Bitterlich, Strand and Prodan, to estimate the number of trees and basal area, in fragments of Ombrophyllous Mixed Forest, in Lebon Régis, SC.

\begin{tabular}{lcccc}
\hline \multirow{2}{*}{ Variável } & \multicolumn{4}{c}{ Métodos } \\
\cline { 2 - 5 } & Área Fixa & Bitterlich & Strand & Prodan \\
\hline $\mathrm{N}($ árv/ha) & $0,34 \mathrm{~ns}$ & $0,30 \mathrm{~ns}$ & $0,48 \mathrm{~ns}$ & $0,47 \mathrm{~ns}$ \\
$\mathrm{G}\left(\mathrm{m}^{2} / \mathrm{ha}\right)$ & $0,36 \mathrm{~ns}$ & $0,97 \mathrm{~ns}$ & $1,72 \mathrm{~ns}$ & $0,66 \mathrm{~ns}$ \\
\hline
\end{tabular}

$\mathrm{N}$ : número de árvores por hectare; G: área basal por hectare; ns: não significativo para teste de $F(\alpha: 5 \%)$, na análise de variância.

Druszcz et al. (2010), na comparação entre os métodos de amostragem de Bitterlich e de Área Fixa com parcela circular em plantios de Pinus taeda, concluíram que o método de Área Fixa com parcela circular foi mais preciso e eficiente para estimativa de diâmetro médio e número de árvores por hectare, e que o método de Bitterlich foi mais preciso e eficiente na estimativa da área basal e volume total.

Já Druszcz et al. (2012), quando compararam os métodos de amostragem de Bitterlich e de Área Fixa em Conglomerado em Cruz, concluíram ser recomendável para estimativa do número de árvores por hectare o método do Conglomerado em Cruz, e para estimativa de diâmetro médio, área basal e volume total, recomenda-se o método de área variável de Bitterlich, pois apresentou maior eficiência e menor custo de levantamento.

\section{CONCLUSÕES}

- Da análise dos resultados, pode-se concluir que o método de Área Fixa apresentou o maior número de espécies florestais e famílias botânicas amostradas. Porém, quando avaliados o número de árvores e área basal por hectare, não foram identificadas diferenças significativas entre os métodos de amostragem.

- O método de amostragem de Strand apresentou-se como o mais eficiente para caracterização do número de árvores e área basal por hectare, porém sem significância estatística com relação aos demais métodos de amostragem testados. 


\section{REFERÊNCIAS}

AGUIAR, O. T. de. Comparação entre os métodos de quadrantes e parcelas na caracterização da composição florística e fitossociológica de um trecho de Floresta Ombrófila Densa no parque estadual "Carlos Botelho" - São Miguel Arcanjo, São Paulo. 120 f. Dissertação (Mestrado em Recursos Florestais) - Escola Superior de Agricultura Luiz de Queiroz, Piracicaba, 2003.

CORDEIRO, J.; RODRIGUES, W. A. Caracterização fitossociológica de um remanescente de Floresta Ombrófila Mista em Guarapuava, PR. Árvore, Viçosa, v. 31, n. 3, p. 545 - 554, 2007.

DIAS, A. C. Composição florística, fitossociologia, diversidade de espécies arbóreas e comparação de métodos de amostragem na Floresta Ombrófila Densa do Parque Estadual Carlos Botelho/SP, Brasil. 184 f. Tese (Doutorado em Recursos Florestais) - Escola Superior de Agricultura Luiz de Queiroz, Piracicaba, 2005.

DIAS, A. C.; COUTO, H. T. Z. Comparação de métodos de amostragem na Floresta Ombrófila Densa Parque Estadual Carlos Botelho/SP - Brasil. Instituto Florestal, São Paulo, v. 17, n. 1, p. 63 - 72, 2005.

DRUSZCZ, J. P.; NAKAJIMA, N. Y.; PÉLLICO NETTO, S.; YOSHITANI JÚNIOR, M. Comparação entre os métodos de amostragem de Bitterlich e de área fixa com parcela circular em plantação de Pinus taeda. Floresta, Curitiba, v. 40, n. 4, p. 739 - 754, 2010.

DRUSZCZ, J. P.; NAKAJIMA, N. Y.; PÉLLICO NETTO, S.; MACHADO, S. A. Custos de inventário florestal com amostragem de Bitterlich (PNA) e conglomerado em cruz (CC) em plantação de Pinus taeda L. Scientia Forestalis, Piracicaba, v. 40, n. 94, 231 - 239, 2012.

FARIAS, C. A.; SOARES, C. P. B.; SOUZA, A. L.; LEITE, H. G. Comparação de métodos de amostragem para análise estrutural de florestas inequiâneas. Árvore, Viçosa, v. 26, n. 5, p. 541 - 548, 2002.

FREESE, F. Elementary statistical methods for foresters. Washington, D.C.: USDA Forest Service, 1967. $87 \mathrm{p}$.

GORENSTEIN, M. R. Métodos de amostragem no levantamento da comunidade arbórea em Floresta Estacional Semidecidual. 92 f. Dissertação (Mestrado em Ciências Florestais) - Escola Superior de Agricultura de Luiz de Queiroz, Piracicaba, 2002.

HERRERA, H. A. R.; ROSOT, N. C.; ROSOT, M. A. D.; OLIVEIRA, Y. M. M. de. Análise florística e fitossociológica do componente arbóreo da Floresta Ombrófila Mista presente na Reserva Florestal Embrapa/Epagri, Caçador, SC, Brasil. Floresta, Curitiba, v. 39, n. 3, p. 485 - 500, 2009.

INSTITUTO BRASILEIRO DE GEOGRAFIA E ESTATÍSTICA (IBGE). Manuais técnicos em geociências: Manual técnico da vegetação brasileira. n. 1. 2 ed. Rio de Janeiro: IBGE, 2012. 275 p.

Manuais técnicos em geociências: Manual técnico de pedologia. n. 4. 2 ed. Rio de Janeiro: IBGE, 2007. $300 \mathrm{p}$.

KURASZ, G. Sistema de Informações Geográficas aplicado ao zoneamento ambiental da Reserva Florestal Embrapa/Epagri, Caçador, SC. 137 f. Dissertação (Mestrado em Ciências Florestais) Universidade Federal do Paraná (Setor de Ciências Agrárias), Curitiba, 2005.

LINGNER, D. V.; OLIVEIRA, Y. M. M.; ROSOT, N. C.; DLUGOSZ, F. L. Caracterização da estrutura e da dinâmica de um remanescente de Floresta com Araucária no Planalto Catarinense. Pesquisa Florestal Brasileira, Colombo, n. 55, p. 55 - 66, 2007.

MARTINS, S. V. Ecologia de florestas tropicais do Brasil. Viçosa: UFV, 2009. 261 p.

MARTINS, D.; RODRIGUES, A. L.; CHAVES, C. L.; MANTOVANI, A.; BORTOLUZZI, R. L. C. Estrutura de um remanescente de Floresta Ombrófila Mista em Urupema, Santa Catarina, Brasil. Revista de Ciências Agroveterinárias. Lages, v. 11, n. 2, p. 126 - 137, 2012. 
MEDEIROS, J. D.; SAVI, M.; BRITO, B. F. A. Seleção de áreas para criação de unidades de conservação na Floresta Ombrófila Mista. Biotemas, Florianópolis, v. 18, n. 2, p. 33 - 50, 2005.

MOSCOVICH, F. A.; BRENA, D. A.; LONGHI, S. J. Comparação de diferentes métodos de amostragem, de área fixa e variável, em uma floresta de Araucaria angustifolia. Ciência Florestal, Santa Maria, v. 9, n. 1, p. 173 - 191, 1999.

MUELLER-DOMBOIS, D.; ELLENBERG, H. Aims and methods of vegetation ecology. New York: John Wiley \& Sons, 1974. 547 p.

NASCIMENTO, A. R. T.; LONGHI, S. J.; BRENA, D. A. Estrutura e padrões de distribuição espacial de espécies arbóreas em uma amostra de Floresta Ombrófila Mista em Nova Prata, RS. Ciência Florestal, Santa Maria, v. 11, n. 1, p. 105 - 119, 2001.

NEGRELlE, R. A. B.; SILVA, F. C. Fitossociologia de um trecho de Floresta com Araucaria angustifolia (Bert.) O. Ktze. no município de Caçador, SC. Boletim de Pesquisa Florestal, Colombo, n. 24/25, p. 37 - 54, 1992.

PÉLLICO NETTO, S.; BRENA, D. A. Inventário Florestal. Curitiba: Editado pelos Autores, 1997. 316 p.

PRODAN, M.; PETERS, R.; COX, F.; REAL, P. Mensura forestal. San José: Instituto Interamericano de Cooperación para la Agricultura (IICA), 1997. 586 p.

SANQUETTA, C. R.; WATZLAWICK, L. F.; DAlla CÔRTE, A. P.; FERNANDES, L. A. V. Inventários florestais: planejamento e execução. Curitiba: Multi-Graphic, 2006. 271 p.

SANQUETTA, C. R.; PIZZATTO, W.; PÉLLICO NETTO, S.; EISFELD, R. L.; FIGUEIREDO FILHO, A. Dinâmica da estrutura horizontal de um fragmento de Floresta Ombrófila Mista no Centro-Sul do Paraná. Ciências Exatas e Naturais, Guarapuava, v. 3, n. 1, p. 43 - 57, 2001.

SEGER, C. D.; DLUGOSZ, F. L.; KURASZ, G.; MARTINEZ, D. T.; RONCONI, E.; MELO, L. A. N. de; BITTENCOURT, S. M. de; BRAND, M. A.; CARNIATTO, I.; GALVÃO, F.; RODERJAN, C. V. Levantamento florístico e análise fitossociológica de um remanescente de Floresta Ombrófila Mista localizado no município de Pinhais, Paraná-Brasil. Floresta, Curitiba, v. 35, n. 2, p. 291 - 302, 2005.

SERVIÇO FLORESTAL BRASILEIRO. Florestas do Brasil em resumo - 2010: dados de 2005-2010. Brasília: SFB, 2010. 152 p.

VANINI, A. Estudo comparativo de dois métodos de amostragem fitossociológica em caixetais (Floresta Ombrófila Densa permanentemente alagada). 120 f. Dissertação (Mestrado em Ciências Florestais) - Escola Superior de Agricultura Luiz de Queiroz, Piracicaba, 1999. 